\title{
Progress in Planar Feature Spatial Reconstruction for Atom Probe Tomography
}

\author{
B. P. Geiser, D.J. Larson, T.J. Prosa and T.F. Kelly
}

CAMECA Instruments, Inc., 5500 Nobel Drive, Madison, WI 53711 USA

The most common reconstruction algorithm for atom probe tomography (APT) data reconstruction has been used with minimal changes for nearly twenty years [1] and has two main limitations: the field evaporated surface is reconstructed as a hemispherical shape (with initial radius R0), and the atomic volume/depth increment is not dependent on the $\mathrm{X}$ or $\mathrm{Y}$ variables. Recently there has been substantial interest in APT reconstruction and the interested reader is referred to two recent reviews [2,3]. In Fig. 1 a field evaporation simulation [4] illustrates the effects of the reconstruction radius on a simple planar interface (no field difference between the two colored regions). A combination of simulated evaporated shape, simulated detector hitmap and experimental data are shown in Fig. 2 for the more probably case where elements with different evaporation fields are present and the evaporated shape is non-hemispherical, Fig. 2a, [5]. The resultant detector hitmap is non-uniform (Fig. 2b) and experimental data of this type, Fig. 2c, exhibit both types of effects observed in Fig. 1.

Methods to deal with these problems include post reconstruction density correction [6,7], methods which operate within the limits of the hemispherical projection [8-10] and methods which remove the hemispherical limitation [11]. The current paper discusses examples of the latter two categories.

The first method discussed is an approach to flattening reconstructed planar features by improving the evolution of reconstruction parameters. We present a technique that imposes planarity by noting that the curvature correction of the first atoms seen at an interface (blue/green arrows in Fig. 3a) must be equal to the integrated change in apex depth when the last atom (red arrow in Fig. 3a) at the interface is detected. With this constraint, and a prior reconstruction, we can correct the radius evolution by scaling the prior tip radius by $x_{R}=\left(d Z_{\text {Apex }} / Z_{p}^{0}\right)^{1 / 3}$. Where $d Z_{\text {Apex }}$ is the accumulated change in tip height over the interface, and $Z_{p}^{0}$ is the curvature correction of the first atom. An example reconstruction applied to interfaces in an AlGaN structure is shown in Fig. $3 \mathrm{~b}$.

One potential method to improve reconstruction, which is not constrained by a hemispherical specimen shape, uses a field evaporation simulation to create a set of transforms to map ions from detector to specimen [11]. Figs. 4a and $4 \mathrm{~b}$ compare the results for a standard reconstruction [1] and the simulation-based method for the case of a low-field-on-high-field bilayer. The larger field of view shown at the interface position in Fig. $4 \mathrm{~b}$ is indicative of the actual blunter specimen shape (lower magnification and thus larger field of view) at this point in the evaporation sequence.

1. $\quad$ P. Bas et al., Appl. Surf. Sci. 87/88 (1995) 298.

2. B. Gault et al., Ultramicroscopy 111(6) (2011) 448.

3. F. Vurpillot et al., Ultramicroscopy (2013) in press.

4. B. P. Geiser et al., Micro. Microanal. 15(S2) (2009) 302.

5. D. J. Larson et al., Ultramicroscopy 111(6) (2011) 506.

6. F. Vurpillot, A. Cerezo and D. J. Larson, Surf. Int. Anal. 36 (2004) 552.

7. F. DeGeuser et al., Surf. Int. Anal. 39 (2007) 268.

8. D. J. Larson et al., J. Microscopy. 243 (2011) 15.

9. F. Vurpillot et al., Ultramicroscopy 111(8) (2011) 1286.

10. B. Gault et al., Ultramicroscopy 111(11) (2011) 1619.

11. D. J. Larson et al., Micro. Microanal. 18(5) (2012) 953. 


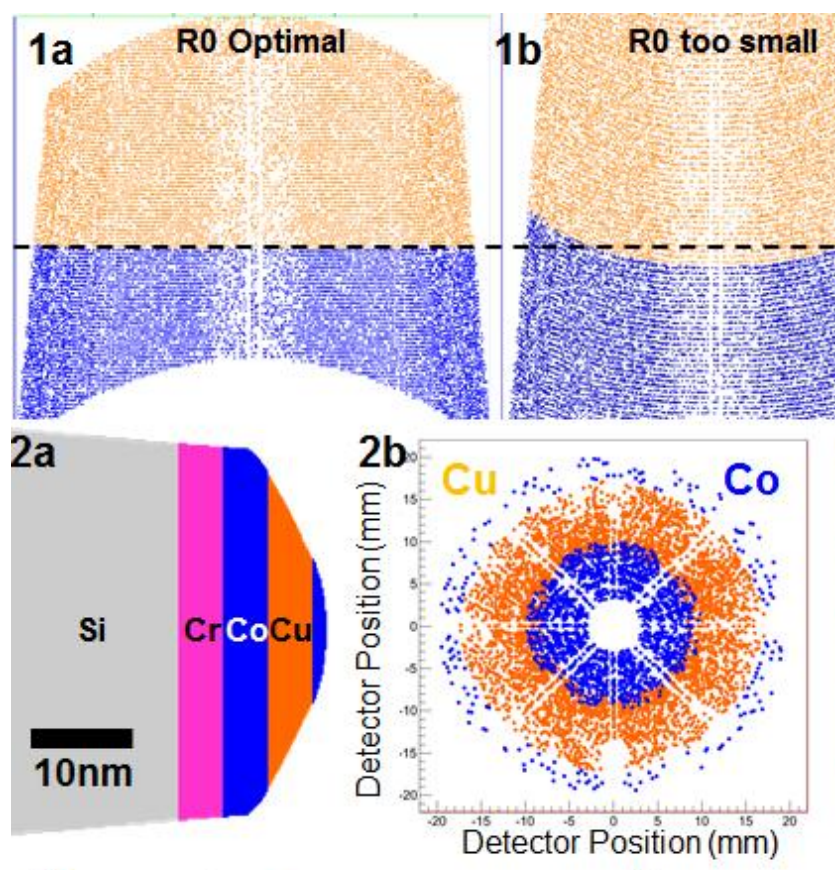

$3 a$

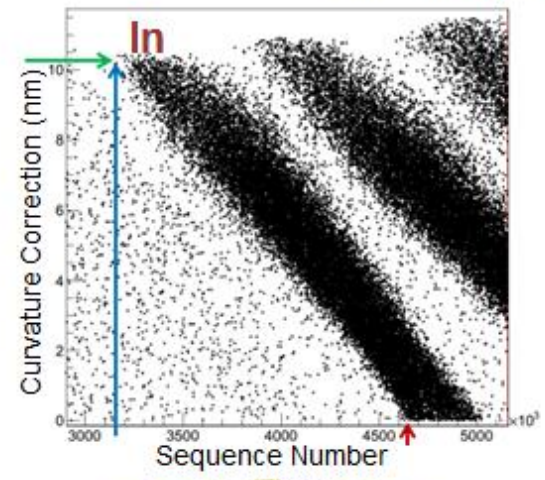

$4 a$

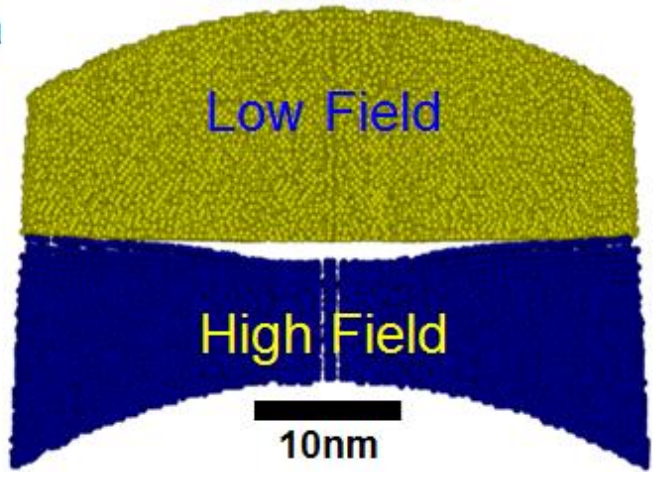

$3 b$

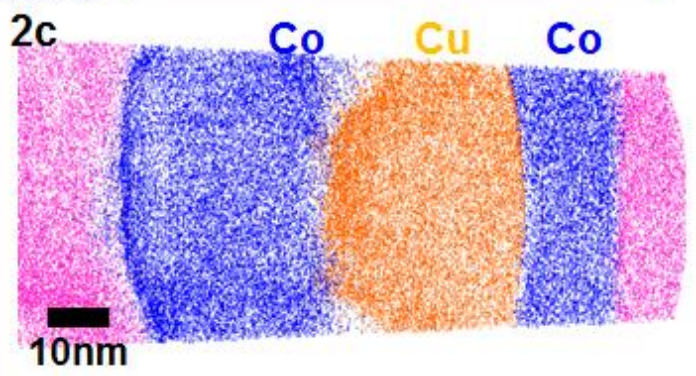

$4 \mathrm{~b}$
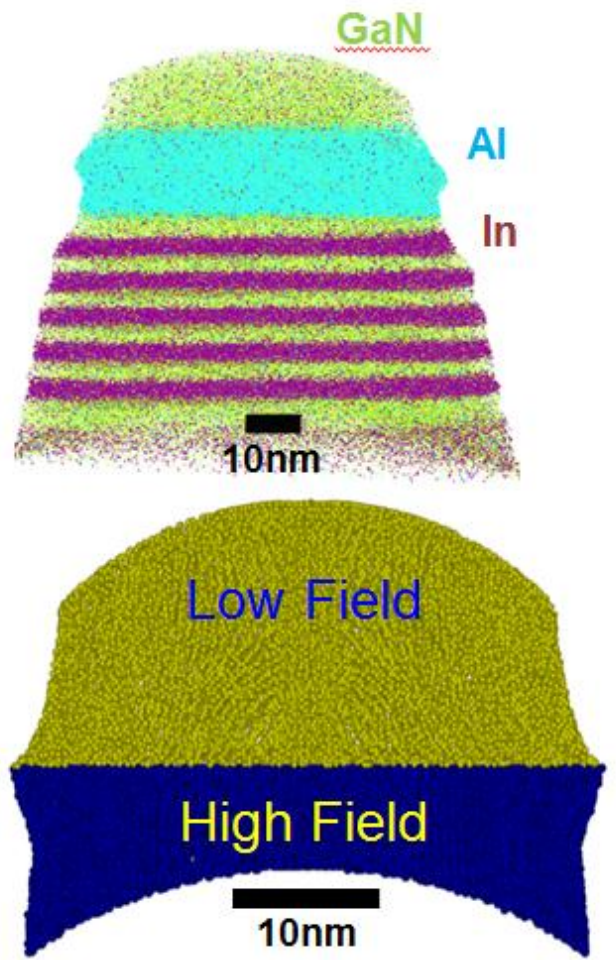

Fig. 1. Field evaporation simulation showing the effects of initial reconstruction radius, R0.

Fig. 2. Field evaporation simulation showing (a) a non-hemispherical evaporated shape, (b) a nonuniform density detector hitmap and (c) experimental data showing the curvatures seen in Fig 1. Fig. 3. (a) Curvature correction of In atoms seen during first-pass reconstruction showing the selected sequence numbers and curvature correction from the first In layer and (b) second pass reconstruction using improved tip radius evolution designed to flatten the layers.

Fig. 4. (a) Standard reconstruction and (b) simulation-based transformation reconstruction. 